\title{
La sociologie urbaine et le marxisme des années 1970 Entretien avec Francis Godard
}

\author{
Antonio Delfini ${ }^{1}$, Janoé Vulbeau ${ }^{2}$ \\ 1. CLERSE - UMR CNRS 8019 \\ 2. ARENES - UMR CNRS 6051
}

Francis Godard est l'auteur dans les années 1970 d'une série d'enquêtes sociologiques sur la France urbaine de la fin des «Trente Glorieuses ». De Rénovation urbaine à Paris (1973), à Crise du logement et mouvements sociaux urbains (1978) en passant par Monopolville (1974), il développe, au sein d'un groupe de chercheurs emmené par Manuel Castells, une approche marxiste visant à dévoiler les contradictions urbaines du mode de production capitaliste.

Cet entretien, mené en décembre 2018, revient sur sa trajectoire personnelle, son parcours intellectuel et aborde tour à tour des considérations politiques, épistémologiques, méthodologiques ainsi qu'un regard singulier sur l'histoire de la sociologie urbaine. Mais au-delà de l'histoire individuelle, il nous renseigne également sur une époque où la recherche urbaine était - pour le meilleur et pour le pire - indissociable des enjeux politiques qui traversaient cette période de bascule pour la société française, à la fois d'installation d'une crise durable mais également d' «âge d'or des luttes » (Matthieu 2010) et d'affirmation progressive d'une gauche de gouvernement.

Pourquoi réaliser cet entretien en 2019 ? À la suite d'un certain nombre de publications qui, sous la forme d'études épistémologiques et historiques (Amiot, 1986 ; Grafmeyer, 2012 ; Pribetich, 2010 ; Lassave, 1997 ; Topalov, 2013), d'entretiens (Pflieger et Castells, 2006 ; Briquet et Préteceille, 1989), ou de retour analytique (Mehl, 1980), voire critique (Garnier, 2010), nous souhaitons donner à voir cette séquence bien particulière qu'a été la sociologie urbaine marxiste à cette époque. À travers cet entretien, nous voulons plus précisément approfondir la question de l'imbrication entre science et politique.

Ayant redécouvert ces travaux dans le cadre de nos propres enquêtes sur l'urbain, nous souhaitons aller au-delà des présentations parfois succinctes, voire caricaturales qui en ont été faites. Cet entretien montre ainsi - à côté de l'engagement politique de ces travaux maintes fois soulevé et, le plus souvent, dénoncé - l'exigence méthodologique et le souci épistémologique qui animaient leurs auteurs. Il revient également sur la structure du champ sociologique de l'époque, dominé par des mandarins (Chombart de Lauwe, Touraine, Lefebvre, Bourdieu), où ces jeunes sociologues - dans leur grande majorité des hommes - devaient être adoubés pour poursuivre une carrière académique, ce qui n'empêchait pas des attaques frontales contre certains de leurs ainés. Il permet également de souligner le caractère collectif des recherches menées et la pluralité des approches et engagements au sein même de la sociologie urbaine marxiste, qui ne peut ainsi être réduit à un cadre théorique unique. Il en souligne enfin certaines limites notamment l'absence de prise en compte d'une perspective de genre ou de « race $»^{1}$ et revient sur la transformation puis l'abandon de ce paradigme dès le milieu des années 1970 par ces auteurs.

\footnotetext{
${ }^{1}$ Sur cette question nous nous permettons de renvoyer à l'un de nos articles en ligne sur le site Métropolitiques voir Vulbeau 2018.
} 

à faire de la sociologie?

Il y a deux raisons : l'une familiale, l'autre plus politique. Il se trouve que je suis issu d'une famille profondément divisée sur le plan sociologique puisque mes grands-parents paternels, qui m'ont élevé, étaient des ouvriers communistes, qui travaillaient à la chaîne en région parisienne. Et du côté de ma mère, c'était des bourgeois royalistes. Donc j'ai été très tôt confronté à deux milieux sociaux radicalement différents, opposés politiquement et étrangers culturellement. Jeune, je vivais chez mes grands-parents communistes mais j'allais au lycée Carnot à Paris, qui était éminemment bourgeois. Je vivais dans le $17^{\mathrm{e}}$ ouvrier et, tous les jours, j'allais au lycée dans le $17^{\mathrm{e}}$ bourgeois. Je passais cette ligne de démarcation qu'était l'avenue de Clichy à l'époque, et je savais qu'en traversant cette rue, je passais d'un monde à l'autre. J'apprenais les normes culturelles : se tenir à table, s'habiller de manière différente, etc. C'était du Bourdieu à l'état pratique. Si, après tout ça, je ne m'étais pas interrogé sur les rapports de classes, ça aurait été un peu bizarre... L'autre raison a trait à mon parcours politique. Je me suis engagé très tôt: j'ai fondé le groupe des jeunesses communistes du lycée Carnot à 13 ans. À l'époque, c'était physique : on se bagarrait avec les mecs du GUD ${ }^{2}$... À Carnot, j'ai eu comme professeur de philosophie Jacques Rancière qui a été pour moi une révélation. Il était major de sa promo à l'École normale supérieure et d'une culture absolument incroyable. On avait un programme officiel : la perception, l'imagination, etc. Mais l'axe de son cours, c'était Nietzsche, Marx et Freud. Intellectuellement, c'était vraiment important pour moi. J'avais un engagement politique mais, lui, va me donner les outils théoriques. Après le bac, je voulais être psychanalyste mais mon père refusait de me payer des études de médecine donc j'ai fait une première année de psychologie à Nanterre. Mais la psycho - travailler sur des rats de laboratoire -, ça m'ennuyait. J'avais une approche à la fois psychanalytique et intellectuellement plus forte. En plus, en sociologie à Nanterre, je voyais Lefebvre, Touraine, Crozier, Baudrillard. Il y avait quand même de sacrées personnalités. Il y avait Fernando Henrique Cardoso qui sera plus tard le président du Brésil... Donc au bout d'un an de psycho, je me suis dit : «c'est pas mon truc ».

Y a-t-il des professeurs, des auteurs ou des textes importants qui ont été pour vous des déclencheurs?

Les lectures, c'était Marx, Marx, Marx. Avec la distinction que constituaient les manuscrits de 1844 entre le jeune Marx et le vieux, j'y reviendrai. Mais l'une des lectures décisives pour la suite de ma carrière de chercheur, c'est l'article : «Y a-t-il une sociologie urbaine ? » de Manuel Castells publié dans la revue Sociologie du travail (Castells, 1968). Après l'avoir lu, je me suis dit : «Là, il se passe quelque chose ». Ça tranchait avec tout ce que j'avais lu auparavant. Ça me paraissait intellectuellement très fort et c'était, idéologiquement, ce qui m'intéressait. À ce moment-là, j'ai eu cette espèce d'intuition qui a fait que je suis devenu ce que je suis. Je vais trouver Castells et je lui

\footnotetext{
${ }^{2}$ Le Groupe d'Union Défense (GUD) - initialement Groupe Union Droit - est un mouvement d'extrême droite né en 1968. Il s'illustre par ses attaques violentes contre les étrangers et les militants d'extrême-gauche. Ses membres participent également aux services d'ordre des campagnes présidentielles de Pompidou (1969), Giscard d'Estaing (1974 et 1981) et Barre (1988).
} 
dis : «je veux travailler avec vous ». Et il accepte. Donc c'est moins un grand texte «classique » qui m'aurait illuminé que la rencontre avec Manuel Castells et la lecture de cet article.

À Nanterre, il y avait aussi Lefebvre qui était marxiste, mais à sa manière : un marxisme des manuscrits de 1844 , ce qui était pour moi un peu fumeux et assez idéaliste. Lefebvre, c'était à la fois le vieux prof de tradition classique en termes académique et le représentant d'un marxisme romantique, c'était le jeune Marx. Alors que moi, j'étais «lutte de classe » : un marxisme à la dure tiré d'Althusser, renforcé par Castells. Les luttes de classe, chez Lefebvre, je les voyais à peine. Pour moi c'était un doux rêveur. Même si, aujourd'hui, en repensant à mes texte je me dis : «quand même, je n'y allais pas de main morte à l'époque... ». Entre Castells et Lefebvre à Nanterre, ça a été frontal, une animosité personnelle. Castells arrivait d'Espagne pour fuir le franquisme. Il était, si ce n'est recherché, du moins, en difficulté politique. Mais Manuel, ce n'était rien, un petit maître de conf', par rapport à Lefebvre. Ce n'était pas une bataille entre mandarins, ils ne jouaient pas dans la même cour. Parce qu'il fallait voir Lefebvre : quand il arrivait dans un amphi, trois cents personnes se levaient et applaudissaient. Lui, traversait la salle en venant de l'arrière avec sa chevelure blanche : c'était très théâtral. Il y avait toujours dix minutes au début de chaque cours où il lisait les courriers qu'on lui envoyait. C'est impensable aujourd'hui, c'était le professeur à l'ancienne... Assez rapidement, Castells va rejoindre Touraine au Centre d'étude des mouvements sociaux (CEMS). Lui et Lefebvre ne sont donc plus en contact institutionnel et ils vont se situer dans des mondes intellectuels complètement différents.

Que s'est-il passé pour vous en 1968 ? Quelles ont été les conséquences de Mai 68 sur votre trajectoire théorique et politique?

Mai 68 a été compliqué pour moi, j'étais très partagé. D'un côté, j'étais à l'Union des étudiants communistes (UEC), mais je n'étais pas complètement à l'aise, il y avait beaucoup d'aspects ringards. De l'autre, avec mon look - cheveux longs, jean douteux et blouson de cuir -, j'étais dans les services d'ordre des gauchos, parce qu'ils considéraient que j'avais le profil et que j'y avais des amis. À côté de ça, je pouvais aussi entrer dans les usines avec mon père et mes grands-parents, bien que je sois en ascension sociale, en train de quitter mon milieu ouvrier d'origine. Donc 68 est un moment de grande confusion pour moi. L'UEC ne me convainquait pas, mais les gauchistes non plus : leur rapport au monde ouvrier était tellement fantasmatique. Je me disais : «comment ces mecs peuvent être l'avant-garde du monde ouvrier alors qu'ils ne savent même pas ce que c'est ? ».

J'ai retrouvé de la cohérence dans l'après-68. À cette époque, il y a eu une sorte de parallèle entre une partie de ce qu'on a appelé «l'école marxiste de sociologie urbaine française » et un courant au sein du Parti communiste français (PCF). Je m'explique : en 1970, je suis redevenu militant communiste parce qu'il y avait le programme commun. Il y avait, au sein du PCF, un petit groupe assez uni avec d'autres sociologues de l'urbain comme Edmond Préteceille, Christian Topalov, etc. 
On était attirés par le parti communiste italien, et Berlinguer ${ }^{3}$ en particulier, car on était, comme lui, anti-soviétique, anti-léniniste, pro-union de la gauche, démocrates. Cette «tendance PCI », c'était une manière d'avoir une certaine ouverture dans les luttes de classes : on était très centrés sur les questions de consommation, de ville, de luttes urbaines. Cette orientation convergeait à la fois sur le plan politique et théorique. À l'époque, dans le milieu sociologique, on était très en vue. On était invités partout, dans tous les colloques. Les gens se plaignaient même de notre omniprésence et du fait qu'on était dominants dans le monde sociologique. Et puis on avait de l'importance politique après avoir organisé des colloques, notamment le grand colloque de Grenoble vers 1973, avec François Ascher ${ }^{4}$. C'est dans ce cadre qu'on a participé à la rédaction de toute la partie «politique urbaine » du Programme commun ${ }^{5}$ à l'époque. Dans cette perspective d'Union de la gauche, on était dans l'optique de créer un nouveau programme de politique du logement. Donc à cette époque, notre pratique de la sociologie était toujours liée à un débouché politique. C'est une séquence qui a un sens pour moi. Dans la foulée du programme commun, j'entre dans cette mouvance du PC qu'on pourrait appeler «pro-Berlinguer » et il va se passer quelque chose qui est en étroite collaboration avec la sociologie urbaine marxiste.

\section{Quels étaient les rapports de pouvoir au sein la sociologie à l'époque?}

C'était un monde de mandarins comme il n'en n'existe plus maintenant. Dans l'institution, la bagarre, c'était Touraine-Bourdieu pour faire passer des candidats à l'École des hautes études en sciences sociales (EHESS), à l'université et au CNRS. Mon recrutement est instructif sur ce point. Nanterre pour Castells et moi, c'est un petit épisode. Je vais très vite et très jeune, à 21 ans, entrer à l'EHESS, boulevard Raspail. Manuel va passer maître-assistant et je vais devenir son chargé de cours. J'ai un bureau juste en face de celui de Touraine, donc je suis en plein en contact avec les mandarins. Chaque année, Touraine soutenait un candidat pour le CNRS. Mais les mandarins se partageaient ces postes : une année Touraine, une année un autre etc. Les candidatures étaient annoncées au mois de janvier et, plusieurs semaines avant, tout le monde se demandait qui Touraine allait choisir comme candidat. Un jour de décembre, il arrive dans mon bureau et me dit : «Godard, vous êtes mon candidat. » C'est comme ça que ça se passait. Au-delà de l'anecdote, ça traduit la façon dont ça se passait : il y avait effectivement des écoles, des écuries et les mandarins se partageaient le marché. C'est paradoxal que Touraine aie soutenu ma candidature. Alors même qu'il savait qui j'étais, qu'il était profondément anti-marxiste et anti-communiste, c'est lui qui m'a soutenu pour que j'entre au CNRS et d'autres après moi. C'est que l'on appelle un démocrate. Cela tient aussi au fait que tout le monde n'est pas tourainien dans le laboratoire et qu'il est obligé de composer. Au contraire de Bourdieu où, si on est dans son laboratoire, on est bourdieusien. Il y a

\footnotetext{
${ }^{3}$ Enrico Berlinguer (1922-1984) a été secrétaire général du parti communiste italien entre 1972 et 1984 . Il se distingue d'autres dirigeants des partis communistes européens en prenant ses distances avec l'URSS, critiquant notamment l'intervention de Prague en 1968, et engage son parti dans une alliance avec le parti démocrate-chrétien italien.

${ }^{4}$ Ce colloque intitulé « Pour un urbanisme », s'est tenu le 6 et 7 avril 1974 à Grenoble. Il a été organisé par l'urbaniste et sociologue François Ascher avec le Parti communiste français et sa revue théorique La Nouvelle Critique.

${ }^{5}$ Le Programme commun de gouvernement est ratifié le 12 juillet 1972 par le parti socialiste et le parti communiste, rejoint ensuite par le mouvement des radicaux de gauche. Dénoncé en 1977, il est néanmoins un jalon essentiel dans la victoire de François Mitterrand à la présidentielle de 1981.
} 
quand même cet échec chez Touraine, c'est qu'il n'entraîne que très peu de monde derrière lui. Il a lancé quelques chercheurs brillants comme Dubet et Wieviorka mais il n'a jamais pu créer une école de sociologie autour de lui. Alors que Bourdieu va entraîner beaucoup de gens sur la base d'un appareil conceptuel assez complexe. Il y a un décalage entre la puissance académique de Touraine, le rôle qu'il va jouer à l'EHESS, et son influence intellectuelle. Ce qui est l'inverse de Bourdieu qui, au CNRS avait peu d'influence (tous ses candidats se faisaient envoyer bouler) alors qu'il avait une très grosse influence intellectuelle et même politique.

\section{Pouvez-vous nous parler de la genèse du « groupe de sociologie urbaine de Nanterre » et de votre premier article collectif intitulé : « rénovation-déportation »?}

À l'origine, Castells regroupe autour de lui quelques étudiants de Nanterre - on était sept ou huit. On signera un article collectif dans Sociologie du travail de ce nom : « groupe de sociologie urbaine de Nanterre ${ }^{6}$. C'est lui qui avait trouvé comme titre, « rénovation-déportation », qui était très fort à l'époque. La violence du terme correspondait à la façon dont il décryptait le social : de manière extrêmement violente. Et puis c'était son sens du théâtre, il voulait frapper les esprits. Manuel était un politique très académique, son monde était celui de l'institution académique, pas celui du militantisme politique. Mais il était plutôt proche des maos ${ }^{7}$, ce qui était très tendance à l'EHESS. Et «déportation», ça sonnait bien mao. Ca reflète donc sa sensibilité politique et son sens du marketing intellectuel. Il se trouve que sur ces sept ou huit étudiants, je suis particulièrement actif, je m'investis beaucoup, et donc il va me proposer de travailler avec lui. Entre 1969 et 1973, je deviens contractuel à l'École des hautes études, et c'est là que je vais travailler avec lui sur Rénovation urbaine puis sur Monopolville. Je suis conscient de tout ce que je lui dois.

\section{Y avait-il un plan général établi dès le départ pour ces recherches ou les terrains et les thématiques ont-ils été trouvés progressivement ?}

Notre ligne de travail, c'était de se situer au cœur des contradictions du capitalisme, de soulever des enjeux structurels. Le choix des terrains était donc très important, il fallait qu'ils aient une capacité démonstrative par rapport à ce qu'on souhaitait prouver. Pour Rénovation urbaine, ce sont les déplacements violents et de grande envergure d'une population ainsi que la question de la ségrégation sociale. On s'y intéresse parce que ce sont des mouvements d'affrontement où on voit se mettre en œuvre la collusion de l'État et des promoteurs... Dunkerque, c'était la ville des monopoles et le lieu de l'exploitation. Lorsqu'on écrit Monopolville, ce n'est pas encore la crise. On est à la fin des Trente glorieuses et donc on a l'impression que la sidérurgie va se développer pendant encore longtemps. C'était des lieux où on pouvait lire ces affrontements. On veut étudier des mouvements de changement de l'espace, de violence des rapports sociaux. Quand on aborde ces

\footnotetext{
${ }^{6}$ Groupe de sociologie urbaine de Nanterre, «Paris 1970 : Reconquête urbaine et rénovation-déportation à Paris », Sociologie du travail, 4, 1970, p. 488-514. Les auteurs de cet article ont été M. Castells, C Cousin, H Delayre, C. Dessane, G. Puig, et C. Skoda.

${ }^{7}$ Sous l'influence de la Révolution culturelle chinoise (1966) et de Mai 68, des groupes de jeunes militants, pour la plupart étudiants à Nanterre et Paris, se rassemblent dans des organisations radicales inspirées des préceptes du président Mao Zedong, comme la GP (Gauche Prolétarienne) ou VLC (vive le communisme). Malgré des différences parfois importantes entre ces courants, ils sont regroupés sous le vocable "maoïstes" ou "maos".
} 
terrains, c'est toujours sous cet angle. Après, il y avait quelque chose d'extrêmement important pour Castells. S'il fallait effectivement se porter sur des endroits stratégiques, il fallait le faire sur la base d'enquêtes empiriques sérieuses, nous devions travailler comme des sociologues et pas des idéologues. Le marxisme en tant que théorie explicative générale ne nous satisfaisait pas. Nous voulions être aussi rigoureux et précis dans nos enquêtes et dans nos méthodes que les autres sociologues non marxistes. On voulait être irréprochables sur le plan professionnel. Dans Rénovation urbaine, ont a fait un gros travail statistique. J'ai passé des heures et des heures à récupérer des données. Il fallait ensuite calculer des coefficients de corrélation, avec - je me rappelle - une vieille Olivetti qui nous sortait des kilomètres de papier. On avait un souci permanent du terrain, quitte à faire le grand écart avec notre dogmatisme théorique. C'était important aussi par rapport aux travaux de Lefebvre où, de notre point de vue, le travail empirique faisait défaut. Nous, ce qu'on disait c'est : « on doit être avant tout des sociologues ».

\section{Il s'agissait pour vous autant de se distinguer des recherches sur l'espace de l'époque que de se positionner par rapport au parti communiste.}

Sans que cela ait été réfléchi à l'avance, Manuel s'est chargé des géographes et moi de Chombart [Chombart de Lauwe]. Avec Rénovation urbaine, on voulait répondre à l'empirisme plat des géographes. On voulait montrer que l'espace se transforme dans l'affrontement de rapports de classes et de domination. Nous entretenions pourtant d'excellentes relations avec les gens de chez Chombart de Lauwe, en particulier avec Henri Coing, qui est d'ailleurs devenu un très grand ami par la suite. Sur le plan intellectuel, on respectait son livre ${ }^{8}$, on trouvait que c'était de la bonne sociologie, que son bouquin était bien construit et que l'enquête était bonne. Mais, pour nous, il n'allait pas au cœur du problème, il était trop culturaliste, il ne voyait pas assez les affrontements. Après, on le retrouvera sur Monopolville puisqu'il va travailler sur Dunkerque et là, Henri va bouger, il va se « dé-chombartiser » et aller plus près de la question des rapports de force ${ }^{9}$. Ceci dit, sa subtilité sociologique interrogeait avec humour notre aplomb structurel.

Chombart, c'est une autre histoire, on est sur un affrontement théorique. C'était un des mandarins de la sociologie urbaine de l'époque, un type important et respectable, une figure de la Résistance. Il était chrétien de gauche et prônait l'alliance avec le PC. Moi, à l'époque, je suis un petit contractuel inconnu de 22 ans et je vais faire un article qui démolit Chombart sur sa théorie des besoins ${ }^{10}$. Je me situais sur un plan théorique en disant que sa théorie des besoins ne tenait pas la route. Elle était pour moi essentialiste : la nature humaine ressent des besoins, on peut les classifier, etc. Or moi, j'étais déjà un peu constructiviste et je disais que les besoins étaient des construits politiques. Chaque époque construit politiquement les besoins auxquels il est important de répondre. En fait, ce qui m'insupportait intellectuellement, c'était cette espèce d'empirisme mou. Alors que, nous, on avait peut-être le défaut inverse : un théoricisme et un dogmatisme dur. Dans le

\footnotetext{
${ }^{8}$ COING Henri, 1966, Rénovation urbaine et changement social, Paris, Éditions ouvrières.

${ }^{9}$ CoING Henri, 1982, La Ville marché de l'emploi, Presses universitaires de Grenoble.

${ }^{10}$ GODARD Francis, 1972, «De la notion de besoin au concept de pratique de classe », La Pensée ${ }^{\circ} 166$, décembre, p. 82 à 108.
} 
monde académique, cet article va créer un petit scandale. Avec Manuel, on se fait de plus en plus aimer par les uns et détester par les autres. Les copains me disent : «t'es fou, Chombart est dans tous ses états. Tu lui es rentré dedans, ça ne se fait pas, tu vas le payer cher. » De l'autre côté, Touraine me félicite, il me sert la main et me dit : «ça, il fallait le faire ! ». Mais il y a également des conséquences politiques directes. C'est intéressant parce que pour moi, cet article n'est pas du tout politique, il est complètement épistémologique. Mais il sort dans La Pensée, une revue proche du PC, et ça a immédiatement un effet politique. À ce moment-là, il y a un rapprochement avec les cathos. Donc je me prends des volées de bois vert de la part de Casanova (PC) ${ }^{11}$ qui était chargé de ce rapprochement. S'en prendre à Chombart, c'était créer un schisme au sein du PC. Donc, voilà, c'est une époque où la théorie avait des effets politiques très directs.

On retrouve ce lien dans Monopolville : Manuel me demande de travailler avec lui, parce qu'il sait que je suis à la commission économique du parti communiste. Vous remarquerez dans la partie économique de ce livre qu'il est fait référence, au début, au capitalisme monopolistique d'État. Ce n'est pas du tout dans le schéma théorique de Manuel, notamment dans la Question urbaine, c'est moi qui importe ça. On fait même un grand écart théorique parce que Manuel soutient la thèse de l'autonomie relative du politique et moi, mon directeur de thèse, c'est Poulantzas, qui a été l'initiateur de cette thèse, qui s'oppose radicalement à celle du capitalisme monopolistique d'État. Donc en fait on démontre le contraire de ce qu'on avance au début. On est en contradiction avec nous-même. Mais ça, en général on le comprend qu'après. On reprend cette thèse du capitalisme monopolistique d'État en disant : « la preuve, Dunkerque en est l'exemple même ». On va se rendre compte de la rigidité et de l'aspect théoriciste de notre démarche. On essaie de le faire rentrer dans le cadre théorique mais ça ne marche pas vraiment.

\section{Pouvez-vous revenir sur le rôle de Manuel Castells et son lien avec le politique ?}

Manuel est fasciné par la politique mais il gravite avant tout dans le monde académique et intellectuel. Je dirais même qu'il s'est cantonné en France à la sphère académique. Il est maître de conférence à l'époque et il veut passer directeur d'étude. D'un côté, il a une grande sensibilité pour les questions politiques et, de l'autre, il est complètement investi dans le monde académique. Il y avait beaucoup de débats à l'époque, notamment avec Poulantzas, autour de la question de l'autonomie relative $\mathrm{du}$ politique et également du rôle de la petite bourgeoisie. Les débats sociologiques étaient surdéterminés par ces grandes questions, je dirais pour le pire et pour le meilleur. Et Manuel avait un fantasme de pouvoir théorique, l'idée qu'il pouvait rendre compte du monde en théorie. N'oublions pas non plus qu'il s'est constitué dans l'internationalisme. Il est à Paris mais il n'est pas Français. La politique française l'intéresse mais il est aussi aux quatre coins du monde. Contrairement à moi où à l'époque j'ai une vision à la fois politiquement centrée sur la France et moins ouverte internationalement. C'est clairement lui qui va porter cette dimension internationale. Sur les questions urbaines, c'est pareil. Il n'était pas vraiment en contact avec les militants, notamment ceux qui luttaient contre la rénovation urbaine. Par contre, ce dont il faut se

\footnotetext{
${ }^{11}$ Laurent Casanova (1906-1972), membre du PCF, chargé des relations avec les intellectuels.
} 
rendre compte, c'est que Castells est un savant doté d'une grande intelligence politique. Si vous regardez qui sont les auteurs de Mouvements sociaux, on est quatre : Dominique Mehl qui est à la Ligue Communiste Révolutionnaire (LCR), Eddy Cherki, qui est maoïste, et moi je suis au PC. On est toujours à la confluence entre le scientifique et le politique, et ça influence aussi nos objets de recherche. Dominique et moi, on va étudier les grands ensembles tandis que Cherki s'occupera des squats.

N'y avait-il pas certains angles morts dans vos recherches? Je pense par exemple à la question des Algériens qui sont évoqués dans Rénovation urbaine comme facteur surdéterminant dans les choix des espaces à rénover sans pour autant que cela fasse l'objet d'un traitement spécifique.

Ça n'était pas tellement une question à l'époque. Il y avait une sorte de cécité des partis de gauche là-dessus. C'était plutôt les mouvements gauchistes qui s'intéressaient aux squats qui trouvaient ces populations. Dans la gauche traditionnelle, cette question ne se posait pas encore. Ce qui se posait c'était la question du sionisme et des Palestiniens. On parlait moins des Algériens. Je dirais que la question palestinienne faisait écran. Il y avait des débats extrêmement violents. Moi je vais retrouver cette question plus tard, notamment concernant les statistiques ethniques. Mais effectivement, je me rappelle que dans Rénovation urbaine, cet indicateur va sortir. C'est significatif car on ne lui attribue pas une importance particulière. Cela ne rentre pas dans notre champ de signification. Je me rappelle de cet indicateur mais qui ne nous trouble pas. On voit bien comment à chaque période, notre perception du monde social est construite. Cela rend aveugle à un certain nombre d'autres questions. Par contre, on va se demander si la rénovation est liée à la transformation de la sociologie électorale. Oui c'est une conséquence, puisqu'on passe de secteurs majoritairement ouvriers à des secteurs dominés par les classes moyennes et supérieures. De là à penser qu'il y aurait une stratégie politique de la part du pouvoir, ça me parait aujourd'hui un peu énorme et même assez stupide.

On a beaucoup parlé pour cette époque d'une recherche radicale mais financée par l'État, qu'en était-il vraiment ? Quels étaient vos rapports avec ces hauts fonctionnaires ?

Ce qui est complètement étonnant, c'est de voir comment l'État finance tout ça. Le ministère de l'Équipement est tenu par les ingénieurs des Ponts et parmi les ingénieurs, il n'y a pas que des gens de droite. Il y avait aussi toute une branche progressiste, plus de gauche. Ce sont des gens comme Michel Conan ${ }^{12}$ ou Pierre Saragoussi ${ }^{13}$. Des gens formidables ! Conan prenait des risques considérables, il fallait que certains rapports restent dans les placards, qu'ils ne soient pas diffusés. Pierre Saragoussi nous commandait un article pour la revue $H$ des HLM. Il savait qui on était. On

\footnotetext{
${ }^{12}$ Michel Conan est paysagiste et urbaniste. Il a été au début des années 1970 le premier responsable de la Mission de la recherche urbaine au ministère de l'Équipement. Il a œuvré pour un rapprochement entre la recherche universitaire et expérimentale, en particulier la sociologie marxiste ainsi que l'équipe du CERFI, et le champ administratif.

${ }^{13}$ Pierre Saragoussi est prêtre et sociologue. Il est au début des années 1970 chargé d'étude à la Fondation de la Recherche Sociale (FORS). Il promeut un agenda chrétien humaniste au sein des politiques urbaines participant notamment à fonder le programme Habitat et Vie Sociale (HVS). Il entre par la suite à L'Union National des HLM, puis à la CDC au début des années 1980.
} 
traitait dans nos articles des rapports propriétaires/ locataires en termes de rapports sociaux. Il y avait des gens qui avaient du courage politique.

Il faut également se rappeler que l'influence de la gauche, qu'elle soit communiste ou socialiste, était très importante à l'époque. Ces fonctionnaires se disaient peut être aussi qu'on pouvait arriver un jour au pouvoir. C'était l'époque du Programme commun. Ils savaient qu'une partie d'entre nous était en train de rédiger la politique du logement de ce programme et qu'il fallait nous ménager. Michel Conan se disait aussi : «voilà des chercheurs qui peuvent nous remuer, qui peuvent nous dire des choses sur notre fonctionnement». Il organisait deux fois par an des séminaires avec des chercheurs de toutes tendances. Un jour il a fait des binômes en mélangeant tout le monde au colloque de Dieppe de $1974^{14}$. Il faut s'imaginer les $\mathrm{DDE}^{15}$ de l'époque. Ils m'avaient mis en binôme avec le DDE de Nice mais il n'est pas venu (il avait dû prendre ses informations auprès des Renseignements Généraux). L'idée de Conan était que si on met des gens très différents tous ensemble, on va pouvoir faire avancer les choses. Je pense que ça en a fait bouger certains. Moi ça m'a fait bouger en me faisant prendre conscience que les hauts fonctionnaires qui représentaient l'État n'étaient pas les brutes technocratiques vendues au capital que nos schémas théoriques nous amenaient à penser. Beaucoup plus tard lorsque je devins élu municipal, j'ai pu, à ce titre, apprécier l'intelligence politique du Préfet Duport qui fréquentait assidûment ces séminaires à sa sortie de l'ENA.

\section{À partir de la fin des années 1970, on a l'impression qu'un mouvement de fond va emporter ce courant de la sociologie urbaine. Comment vivez-vous ces transformations dans vos travaux sociologiques et dans votre engagement politique?}

À partir de 1975-1976, il y a effectivement un basculement complet. Au-delà de la sociologie ou de la politique, c'est un basculement culturel et idéologique général que j'ai du mal à expliquer. Ce mouvement a pour moi des conséquences épistémologiques et politiques profondes. Après Monopolville et Les mouvements sociaux, Manuel part aux États-Unis et devient professeur à Berkeley vers 1976. Personnellement, j'opte pour d'autres perspectives de recherche. J'étais un peu las des grands schémas et du théoricisme. L'approche hyper-théorique et descendante commençait à m'insupporter. Tout en restant convaincu de l'analyse en termes de rapports sociaux, je sors des problématiques urbaines structurelles $\mathrm{Au}$ lieu d'attribuer de manière théorique des «intérêts de classe » à chaque individu comme nous le faisions dans l'ouvrage sur les mouvements sociaux urbains, je cherche à comprendre comment les individus construisent leur vie. Je ne deviens pas bourdieusien, mais les thèmes de recherche, les questionnements sur la famille ou les modes de vie vont occuper plus de place. De mes travaux précédents, je garde le sujet du pouvoir local mais je le prends par un autre bout, je vais plutôt questionner le concept de représentation politique. Sans rompre complètement, j'ai changé de perspective. Mais il y a effectivement un retournement

\footnotetext{
${ }^{14}$ Les actes ont été publiés sous le titre : Politiques urbaines et planification des villes. Actes du colloque de Dieppe, 89-10 avril 1974, Paris : Commissariat général au Plan et Mission de la Recherche au ministère de l'Équipement.

${ }^{15}$ Les Directions Départementales de l'Equipement (DDE) sont créées en 1967. Ce sont les services déconcentrés du ministère de l'Equipement dans les différents départements français.
} 
épistémologique important à l'époque avec des revues qui disparaissent, d'autres qui se créent, etc. Pour moi, le pire, c'est de rester prisonnier de grilles d'analyse trop fermées et le meilleur, c'est de conserver une vision structurelle des choses.

Sur le plan politique, l'éclatement du Parti communiste provoque un autre basculement ${ }^{16}$. Cela correspond à la reprise en main du parti par Georges Marchais à la fin des années 1970 et, en particulier, à son discours de Kaboul qui justifie l'invasion de l'Afghanistan par les soviétiques. Des tensions très fortes sont apparues à ce moment-là. Et lorsque, lors d'un congrès vers 1979, Marchais a posé la grande question du «bilan globalement positif », c'était pour faire le tri au sein du Parti. Parce qu'il sentait qu'au sein du PC, ça commençait à bouger. Moi, à l'époque, je suis à Nice au comité fédéral du PC. Je faisais partie de ceux qui disaient : «il n'y a pas de bilan globalement positif ». Et j'ai bien vu comment ça s'est passé : c'était une manière d'identifier ceux qui étaient dangereux à l'intérieur et de les circonscrire. Tous ceux qui avaient mobilisé beaucoup de forces intellectuelles, et en particulier notre groupe de sociologues qui avait joué un rôle dans l'élaboration de la politique urbaine du Programme commun, cette espèce d'intellectuel collectif que l'on formait au sein du Parti, tout ça a disparu. Je quitte le PC à ce moment-là et bien d'autres aussi. Ensuite, mon histoire politique, c'est que je me «social-démocratise» en quelque sorte. Je deviens compagnon de route du PS dans les années 1980 et j’y entre officiellement au début des années 1990. En 1981, l'arrivée de la gauche au pouvoir est, pour moi et beaucoup d'autres, fantastique. J'habite à Nice, dans un coin particulièrement réac', donc pour moi et tous les gens de gauche, c'est énorme. Ce n'est qu'ensuite qu'on va découvrir peu à peu les joies du mitterrandisme. Moi, je me sentais plus proche de Michel Rocard: le Rocard qui s'appuie sur le monde associatif, qui va chercher des ressources dans la société civile. Donc je vois d'un mauvais œil Rocard se faire malmener par Mitterrand. Mais j'adhère au PS pour ça : c'est un parti où des courants divers peuvent exister. C'est sûr, ce parcours politique influence aussi ma façon de faire de la sociologie.

\section{Comment vous vivez ce changement au sein de l'institution? L'arrivée au pouvoir de la gauche a-t-elle entraîné de nouveaux financements pour la sociologie?}

1981 va changer beaucoup de choses. À partir de ce moment-là, quand on va dans les ministères, on a des hommes de gauche - et qui n'ont pas à se cacher - en face de soi, ce qui change la donne. Il y a effectivement eu des relances au niveau du financement ministériel. En 1989, Rocard, en tant que Premier ministre, met de l'argent de côté pour engager des programmes de recherche sur les quartiers dits «en difficulté ». Moi, je suis de retour des États-Unis où j'ai été professeur à Stanford pendant un an et demi et quand je reviens, on me demande d'assumer la co-responsabilité du Programme interdisciplinaire de recherche sur la ville, le Pir-Ville ${ }^{17}$, avec Gabriel Dupuy, directeur de ce programme. J'arrive au mois de septembre au CNRS et le directeur général nous dit : « il faut engager le financement de Rocard avant le mois de décembre sinon il retourne au pot commun. » Donc très rapidement, nous mettons un programme en place pour affecter le financement. On a fait

\footnotetext{
${ }^{16}$ Sur cette période de déclin du parti communiste voir Pudal 2009.

${ }^{17}$ Ce programme de recherche avait pour but de faciliter les coopérations entre disciplines et de développer les études internationales.
} 
travailler des ingénieurs, des médecins et des sociologues et bien d'autres. Tous les grands laboratoires de sociologie y ont participé. Mais la sociologie urbaine perd de plus en plus son caractère critique, elle devient de plus en plus empiriste, elle n'embraye plus comme auparavant sur de grandes visions d'ensemble de notre société. Ce premier regain de financement s'est poursuivi par le programme de recherche sur la ville du ministère de la Recherche, dont j'ai été directeur dans la deuxième partie des années 1990. J'ai également été président d'un groupe de prospective de la DATAR sur les temporalités sociales qui avait déjà bien pointé des questions essentielles comme celle de la mobilité, celle des rapports entre aménagement du temps et aménagement de l'espace ou celle des effets du développement des NTIC sur l'organisation de l'espace. Intellectuellement, la question urbaine n'occupait plus la place qu'elle avait dans le champ intellectuel des années 1970. C'est le moment de l'apparition de la question environnementale. À partir de la fin des années 1990, la recherche sur la ville va peu à peu céder la place à la question du développement durable, qui prendra le relai dans la capacité de la recherche à se porter sur les enjeux cardinaux de notre société. Lorsque je la regarde depuis le XXIème siècle, la recherche urbaine marxiste du début des années 1970 et les débats qui l'ont entourée m'apparaissent comme un objet intellectuel un peu exotique. Ne peut-on dire la même chose de cette époque? 


\section{Bibliographie}

AMIOT Michel, 1986, Contre l'Etat, les sociologues. Éléments pour une histoire de la sociologie urbaine en France, 1900-1980, Paris, Éditions de l'École des hautes études en sciences sociales.

CASTELLS Manuel, 1968, «Y a-t-il une sociologie urbaine ?», Sociologie du travail, 10e année ${ }^{\circ} 1$, Janvier-mars, p. 72-90.

CASTELLS Manuel, CHERKI Eddy, GODARD Francis et MEHL Dominique, 1978, Crise du logement et mouvements sociaux urbains, Paris, Mouton.

CASTELLS Manuel et GODARD Francis, 1974, Monopolville. Analyse des rapports entre l'entreprise, l'Etat et l'urbain à partir d'une enquête sur la croissance industrielle et urbaine de la région de Dunkerque, Paris, Mouton.

GARNIER Jean-Pierre, 2010, Une violence éminemment contemporaine. Essais sur la ville, la petite bourgeoisie intellectuelle et l'effacement des classes populaires, Marseille, Agone, coll. Contre-Feux.

GODARD Francis, 1972, «De la notion de besoin au concept de pratique de classe », La Pensée n 166, décembre, p. 82 à 108.

GODARD Francis (dir.), 1973, La rénovation urbaine à Paris. Structure urbaine et logique de class, Paris, ed. Mouton.

GRAFMEYER Yves, 2012, "La sociologie urbaine dans le contexte français », SociologieS [En ligne], Dossiers, Actualité de la sociologie urbaine dans des pays francophones et non anglophones, mis en ligne le 15 novembre 2012, consulté le 29 mai 2019.

GROUPE DE SOCIOLOGIE URBAINE DE NANTERRE, 1970, «Paris 1970 : Reconquête urbaine et rénovation-déportation à Paris », Sociologie du travail, 4, 1970, p. 488-514.

LASSAVE, Pierre, 1997, Les sociologues et la recherche urbaine dans la France contemporaine, Toulouse, Presses Universitaires du Mirail.

MATHIEU Lilian, 2010, Les années 70, un âge d'or des luttes ?, Paris, Textuel "Petite encyclopédie critique ».

MEHL Dominique, 1980, «les voies de la contestation urbaine », les Annales de la recherche urbaine, $\mathrm{n}^{\circ} 6$, p. 26-62.

PUDAL Bernard, 2009, Un monde défait. Les communistes français de 1956 à nos jours, Pari, Éditions du Croquant, coll. «Savir/Agir ».

PFLIEGER Géraldine, 2006, De la ville aux réseaux : dialogue avec Manuel Castells, Lausanne, Presses polytechniques et universitaires romandes.

PRETECEILLE Edmond et BRIQUET Jean-Louis, 1989, «Les marxistes et la question urbaine », Politix, vol. 2, n`7-8, Octobre-décembre. L'espace du local. pp. 24-29.

PRIBETICH Justine, 2010, La Sociologie urbaine en France : constitution et évolution d'un domaine de recherche spécialisé, thèse de doctorat de sociologie sous la direction de Bernard Valade, université Paris-5 Descartes.

TOPALOV Christian, 2013, « Trente ans de sociologie urbaine. Un point de vue français », Métropolitiques, 16 octobre 2013. URL : http://www.metropolitiques.eu/Trente-ans-de-sociologie-urbaine.html.

VULBEAU Janoé, 2018, "Les « Nord-Africains » dans la rénovation urbaine des années 1960 », Métropolitiques, 31 mai 2018. URL : https://www.metropolitiques.eu/Les-Nord-Africains-dans-larenovation-urbaine-des-annees-1960.html 